Trauma Berufskrankh 2006 • 8[Suppl 2]:

S171-S177

DOI 10.1007/s10039-006-1101-7

Online publiziert: 21. März 2006

(c) Springer Medizin Verlag 2006

\author{
R. Braunschweig ${ }^{1} \cdot$ O. Schilling ${ }^{1} \cdot$ I. Höller ${ }^{1} \cdot$ W. Wawro ${ }^{2}$ \\ ${ }^{1}$ Klinik für bildgebende Diagnostik und Interventionsradiologie, \\ BG-Kliniken Bergmannstrost, Halle, Saale \\ ${ }^{2}$ Klinik für Unfall- und Wiederherstellungschirurgie, \\ BG-Kliniken Bergmannstrost, Halle, Saale
}

\section{Bone bruise und MRT}

\section{Klinischer Stellenwert eines diagnostischen Zeichens}

Bei der Betrachtung von ossären, ligamentären, kapsulären und sonstigen Weichteilveränderungen steht neben Entzündungen und muskuloskelettalen Tumorerkrankungen v. a. das „Trauma“ als wesentlicher behandlungspflichtiger Gesamtkomplex im Vordergrund.

Diagnostische Herausforderung ist bei der Erstbetreuung von muskuloskelettalen Verletzungen der Nachweis eines manifesten Organschadens bei auffälliger Klinik (Schmerzen, functio laesa), jedoch fehlendem Befund im projektionsradiographischen Untersuchungsspektrum (Röntgen, s. $\square$ Tab. 1). Im Rahmen der bildgebenden Diagnostik hat die Magnetresonanztomographie bei der Erstbeurteilung und Verlaufskontrolle (inklusive Begutachtung) muskuloskelettaler Ver- letzungen wesentliche Bedeutung bei der Differenzierung bzw. dem Ausschluss auch geringer ossärer Verletzungsmuster erlangt. Herausragendes Merkmal ist die hoch sensitive Nachweisrate von (z. B. röntgenologisch okkulten) Verletzungsmustern, z. B. von Bone bruise. Grundlage hierfür bilden Ödematisierung und Hämatombildung in den jeweiligen Kompartmenten (z. B. Knochen).

\section{Definition}

Die Bone bruise äußert sich als hyperintense Signalveränderung in T2-gewichteten magnetresonanztomographischen Untersuchungssequenzen mit diffuser oder lokalisierter Ausbreitung im Knochen [5]. Es handelt sich nicht um ei- ne Fraktur, Grundlage der Bone bruise sind vielmehr Wasser- bzw. Blutansammlungen. Sie ist von Mikrofrakturen und/ oder okkulten Frakturen abzugrenzen (• Abb. 1, 2). Sie ist keine „übersehene Fraktur“(• Abb. 3).

Die Bone bruise bedarf einer exakten klinischen und anamnestischen Einordnung. Beim Fehlen weiterer Verletzungsfolgen (z. B. additive Frakturen usw.) erklärt sie ggf. Schmerzsituationen und kann als gutachterlicher Ausgangsbefund gewertet werden.

Das diagnostische Zeichen „Bone bruise“ definiert seinen klinischen Stellenwert vorrangig aus der Konstellation „auffällige Klinik und leeres Röntgenbild“. Die Bone bruise ist als ein MR-morphologisches Bindeglied zwischen auffäl-

\begin{tabular}{|c|c|c|c|c|}
\hline Verfahren & $\begin{array}{l}\text { Physikalisches Prinzip der } \\
\text { Bilderzeugung }\end{array}$ & Vorteile & Nachteile & Besonderheiten \\
\hline \multirow[t]{3}{*}{ Röntgen (Projektionsradiographie) } & \multirow[t]{3}{*}{ Ionisierende Strahlung } & Schnell, billig & Strahlenbelastung & $\begin{array}{l}\text { Hohe Detailerkenn- } \\
\text { barkeit }\end{array}$ \\
\hline & & Flächendeckend verfügbar & Überlagerungen & \multirow{2}{*}{$\begin{array}{l}\text { Konventionelle } \\
\text { Tomographie }\end{array}$} \\
\hline & & Reproduzierbar & $\begin{array}{l}\text { Eingeschränkte Weich- } \\
\text { teilinformation }\end{array}$ & \\
\hline \multirow[t]{2}{*}{$\mathrm{CT}$} & \multirow[t]{2}{*}{ Ionisierende Strahlung } & Überlagerungsfreie Darstellung & \multirow[t]{2}{*}{ Strahlenbelastung } & $\begin{array}{l}\text { Hoher Qualitäts- } \\
\text { standard }\end{array}$ \\
\hline & & 3D-Rekonstruktionen & & 3. Ebene \\
\hline \multirow[t]{2}{*}{ MRT } & \multirow[t]{2}{*}{ Hochfrequenzfelder } & Hervorragender Weichteilkontrast & Störanfällig & Hohe Sensitivität \\
\hline & & Keine Schädigungen bekannt & $\begin{array}{l}\text { Cave: z. B. Metallim- } \\
\text { plantate }\end{array}$ & $\begin{array}{l}\text { Teilweise hohe } \\
\text { Spezifität }\end{array}$ \\
\hline \multirow[t]{3}{*}{ Sonographie } & \multirow[t]{3}{*}{$\begin{array}{l}\text { Darstellung von Impedanz- } \\
\text { unterschieden }\end{array}$} & Schnell & Untersucherabhängig & $\begin{array}{l}\text { Weichteilbefunde } \\
\text { (z. B. Sehnen) }\end{array}$ \\
\hline & & Kostengünstig & \multirow{2}{*}{$\begin{array}{l}\text { Schlecht reprodu- } \\
\text { zierbar }\end{array}$} & \multirow{2}{*}{$\begin{array}{l}\text { Beliebige Schnitt- } \\
\text { orientierung }\end{array}$} \\
\hline & & Keine Schädigungen bekannt & & \\
\hline
\end{tabular}




\section{Bone bruise}
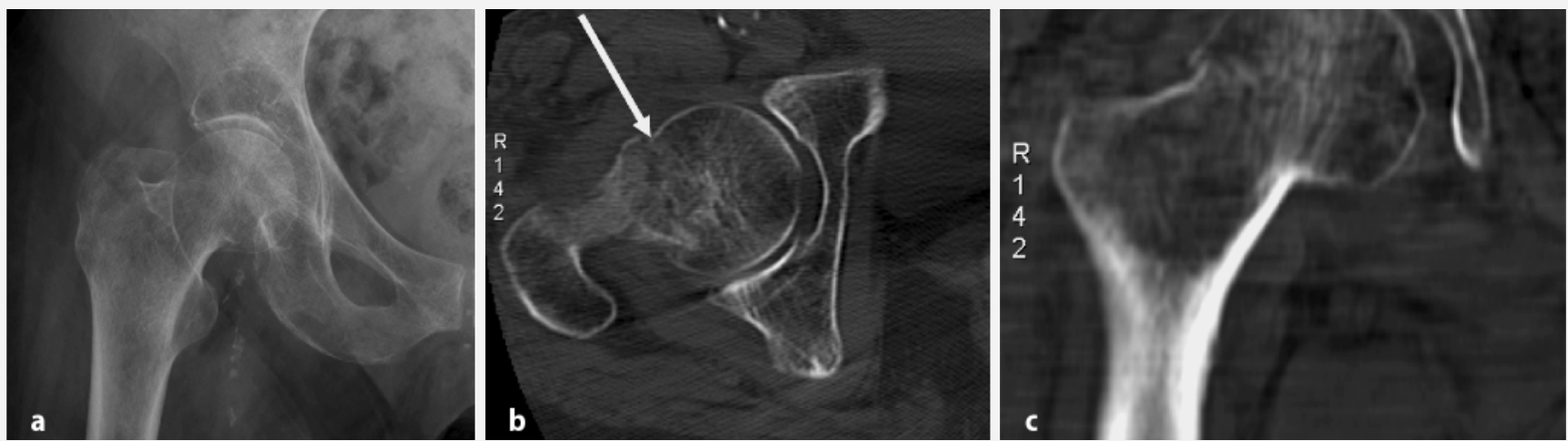

Abb. $1 \Delta$ Differenzialdiagnose Mikrofraktur: röntgenologisch (a) kein (eindeutiger) Frakturnachweis, computertomographisch (b, Pfeil) Nachweis einer mäßig dislozierenden medialen Schenkelhalsfraktur; koronare Rekonstruktion (c): Nachweis der medialen Schenkelhalsfraktur rechts, keine Bone bruise sondern computertomographisch (und projektionsradiographisch?) fassbare Mikrofraktur
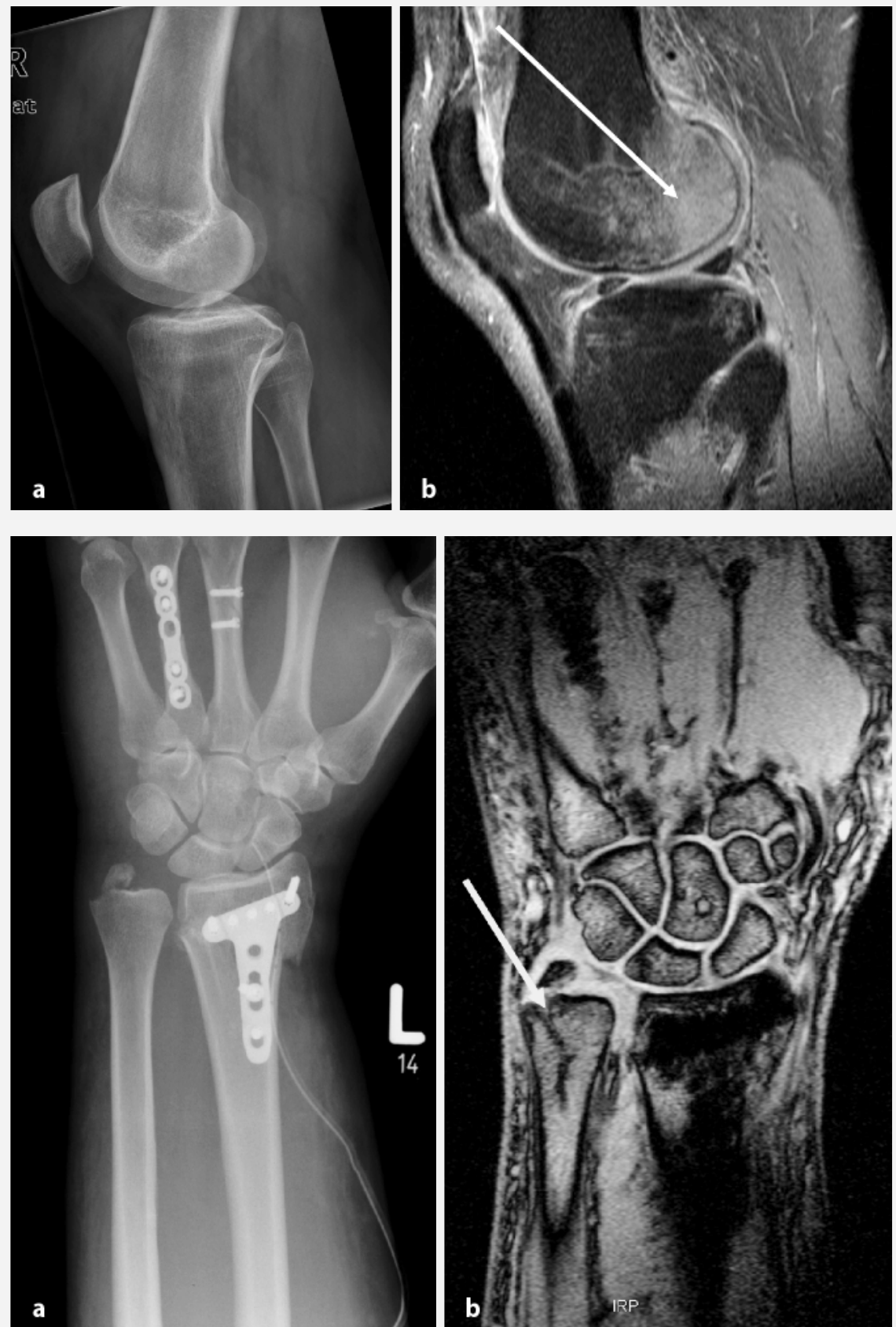

Abb. 24 MRT-Befund: auffällige Klinik vs.,leeres" Röntgenbild: Nachweis einer landkartenartigen Bone bruise am dorsalen Femurkondylus (Pfeil), Zeichen einer morphologisch fassbaren Verletzung (b), röntgenologisch unauffälliger Befund (a), keine Fraktur

Abb. 34 Differenzialdiagnose okkulte Fraktur: röntgenologisch (a) Nachweis der osteosynthetisch versorgten, typischen, (s. Abriss des Processus styloideus ulnae) distalen Radiusfraktur, magnetresonanztomographisch (b) Nachweis einer zusätzlichen Fraktur in der distalen Ulnametaphyse (Pfeil), damit Nachweis einer (projektionsradiographisch) okkulten Fraktur in der Ulna, einer anderen Klassifikation (distale Unterarmfraktur) entsprechend, Befunde sind gegenüber Bone bruise abzugrenzen 
ligem klinischem Befund und projektionsradiographisch okkulter Bildgebung zu verstehen!

Klinische Konsequenzen sind nicht allein aus dem Befund „Bone bruise“ abzuleiten. Diese Diagnose determiniert in der Regel eher ein konservatives Behandlungskonzept.

\section{Diagnosetechnik}

Die Projektionsradiographie (klassisches Röntgen) hat sich als wesentliche Bildgebung bei ossären Verletzungen bewährt. Das Verfahren ist überall verfügbar, kostengünstig und sichert standardisierte Technik und Qualität.

Neben wenigen indirekten Zeichen (z. B. Humeruskopfhochstand, Radiuskopfsubluxation, Gelenkaufklappbarkeit usw.) sind jedoch additive, ligamentäre, kapsuläre oder sonstige Weichteilverletzungen nur unsicher bewertbar. Im Gegensatz zur Projektionsradiographie ist die Magnetresonanztomographie (MRT) als Schnittbildverfahren und aufgrund der anderen bildgebenden Technologie (- Tab. 1) in der Lage, additiv zum Röntgenbild intraossäre und ligamentäre bzw. Weichteilverletzungen sensitiv nachzuweisen [1]. So können z. B. mit einer Sensitivität von $96 \%$ durch spezielle fettgesättigte, $\mathrm{T}_{2}$-gewichtete Spinechosequenzen (STIR-Sequenz) ein treffsicherer Nachweis bzw. Ausschluss von intraossären Verletzungsmanifestationen (z. B. Bone bruise, Mikrofrakturen) geführt werden.

\section{Substrat und Bildgebung}

Pathophysiologisch-anatomisches Substrat des magnetresonanztomographischen Nachweises von intraossären Veränderungen sind Protonendichteverschiebungen im jeweils dargestellten Zielorgan. Diese werden durch Wassereinstrom (Ödem) und/oder Hämatombildung (Blut und Abbauprodukte) bewirkt (- Abb. 4).

Bereits frühe Publikationen zur MRTDiagnostik haben auf den klinischen Stellenwert und die Definition [5] der Bone bruise aufmerksam gemacht. Ödem- oder Hämatombildung durch andere Ursachen (Entzündungen, Tumoren usw.) sind nicht als Bone bruise zu bezeichnen.

Trauma Berufskrankh 2006 · 8[Suppl 2]: S171-S177 DOI 10.1007/s10039-006-1101-7

○) Springer Medizin Verlag 2006

\section{R. Braunschweig $\cdot 0$. Schilling $\cdot$ I. Höller $\cdot$ W. Wawro Bone bruise und MRT . Klinischer Stellenwert eines diagnostischen Zeichens}

\section{Zusammenfassung}

1. Ossäre und Weichteilverletzungen bedürfen einer subtilen Diagnostik mittels bildgebender Verfahren. Differenzierte Therapieansätze stehen zur Verfügung.

2. Als Basis steht die Projektionsradiographie in 2 Ebenen zur Verfügung. Ein im Hinblick auf Anamnese, Unfallhergang und klinischem Beschwerdebild adäquater Befundnachweis beendet in aller Regel die bildgebende Diagnostik.

3. Computertomographische Verfahren kommen als ergänzende Basisdiagnostik zur Beantwortung operationstaktischer Fragen zum Einsatz.

4. Bei diskrepanter Befundkonstellation zwischen auffälligem klinischem Befund, jedoch leerem Röntgenbild, folgt unmittelbar die Kernspintomographie zum Ausschluss von Mikrofrakturen bzw. Bone bruise.

5. Die Bone bruise ist eine an ein Trauma gebundene Ödem- oder Hämatombildung intraossär. Diese kann hoch sensitiv kernspintomographisch erfasst werden. Die Bone bruise ist gegenüber Mikrofrakturen oder okkulten Frakturen im Sinne der trabecularen Zerstörung abzugrenzen und mit diesen nicht gleichzusetzen.

6. Untersuchungstechnisch sind sowohl im Gelenk- als auch im Stammskelettbereich T1- und T2-gewichtete sowie STIR- und SPIR-orientierte Sequenzen einzusetzen. Es ist auf eine möglichst dreidimensionale orthogonale Darstellung zu achten.

7. Der Nachweis der Bone bruise ergibt kein aktives chirurgisches Vorgehen. Die Bone bruise kann jedoch den Verletzungsmechanismus insgesamt einordnen, die Befundobjektivierung ergänzen und ggf. konservative Therapieansätze oder mittelfristige Begutachtungen determinieren. Bei kernspintomographisch fehlendem Nachweis einer Bone bruise ist eine Verletzung auszuschließen.

\section{Schlüsselwörter}

Ossäre Verletzungen - Weichteilverletzungen . Auffällige Klinik - leeres Röntgenbild · Bone bruise - Diagnosealgorithmus

\section{Bone bruise and MRI . Relative value of a diagnostic sign in clinical practice}

\section{Abstract}

Bony and soft tissue injuries require precise diagnosis by means of imaging techniques. The basic examination performed is projection radiography in two planes. If the findings yielded by this technique appear to bear out expectations raised by the history, the mechanism of injury and the clinical examination, no further imaging procedures need be carried out. CT may be helpful when decisions have to be made on any surgery to be performed. When there is a discrepancy in the findings (e.g. suggestive clinical findings and unremarkable roentgenogram) MR imaging should be performed to check for microfractures and/or bone bruising. The latter takes the form of intraosseous oedema or haematoma linked with a trauma, and it is important to differentiate it from microfractures or occult fractures. Both T1- and T2-weighted sequences are needed, as are STIR- and SPIRoriented sequences; as far as possible, the images must be three dimensional and orthogonal. Detection of bone bruising does not generally mean that active surgical intervention is indicated, but it does help to classify the mechanism of injury and complement the objectivization of the findings; it is also a significant factor in selection of the conservative treatment approach to be adopted or for a medium-term expert assessment.

\section{Keywords}

Bony injuries · Soft tissue i njuries · Suggestive clinical findings - Unremarkable roentgenogram · Bone bruising · Algorithmus for diagnosis 


\section{Bone bruise}
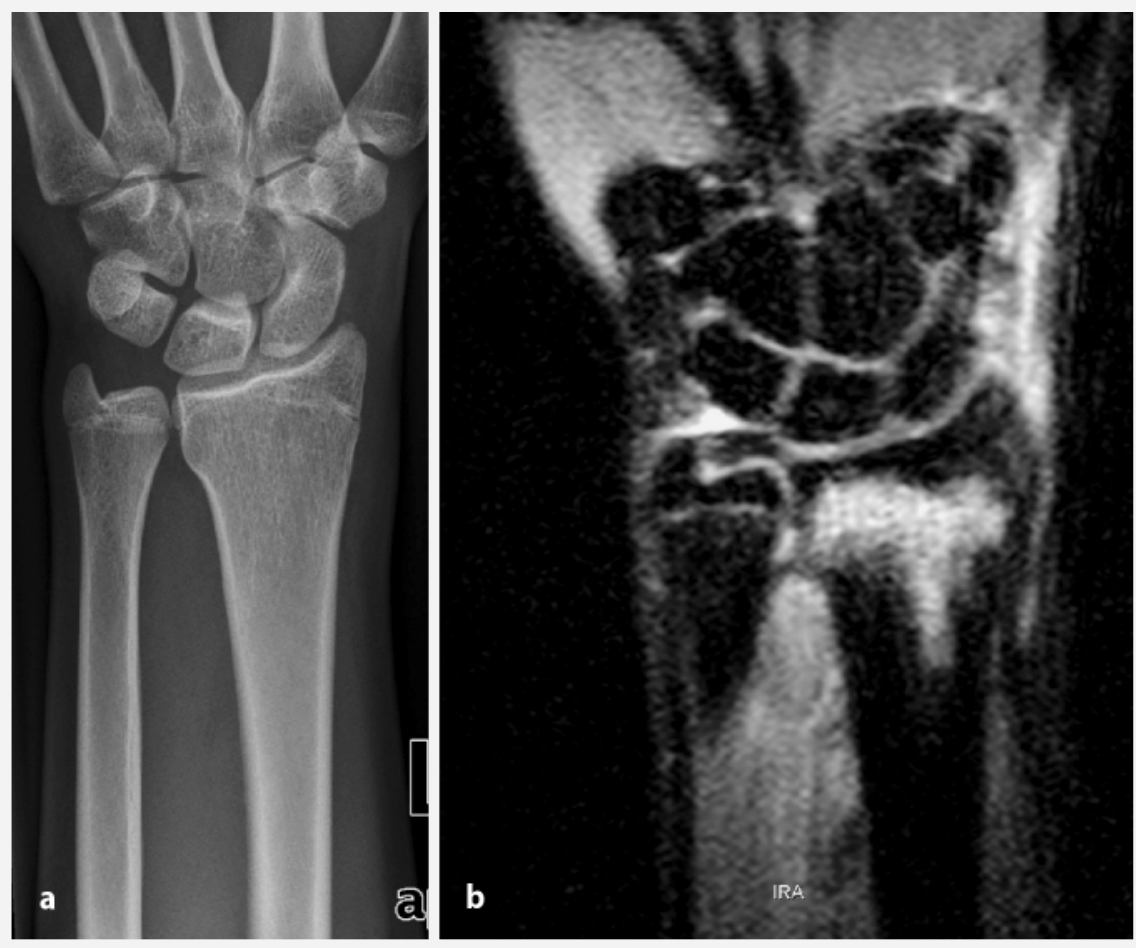

Abb. $4<$ Definition Bone bruise:Vergleich von a.-p. Röntgenaufnahme (a) und koronarer STIRSequenz (b) (MRT), röntgenologisch unauffälliger Befund, magnetresonanztomographisch erheblicher Densitätsanstieg einer geografisch metaepiphysären Läsion im Bereich des distalen Radius, Befund entspricht Bone bruise (am ehesten Einblutung)
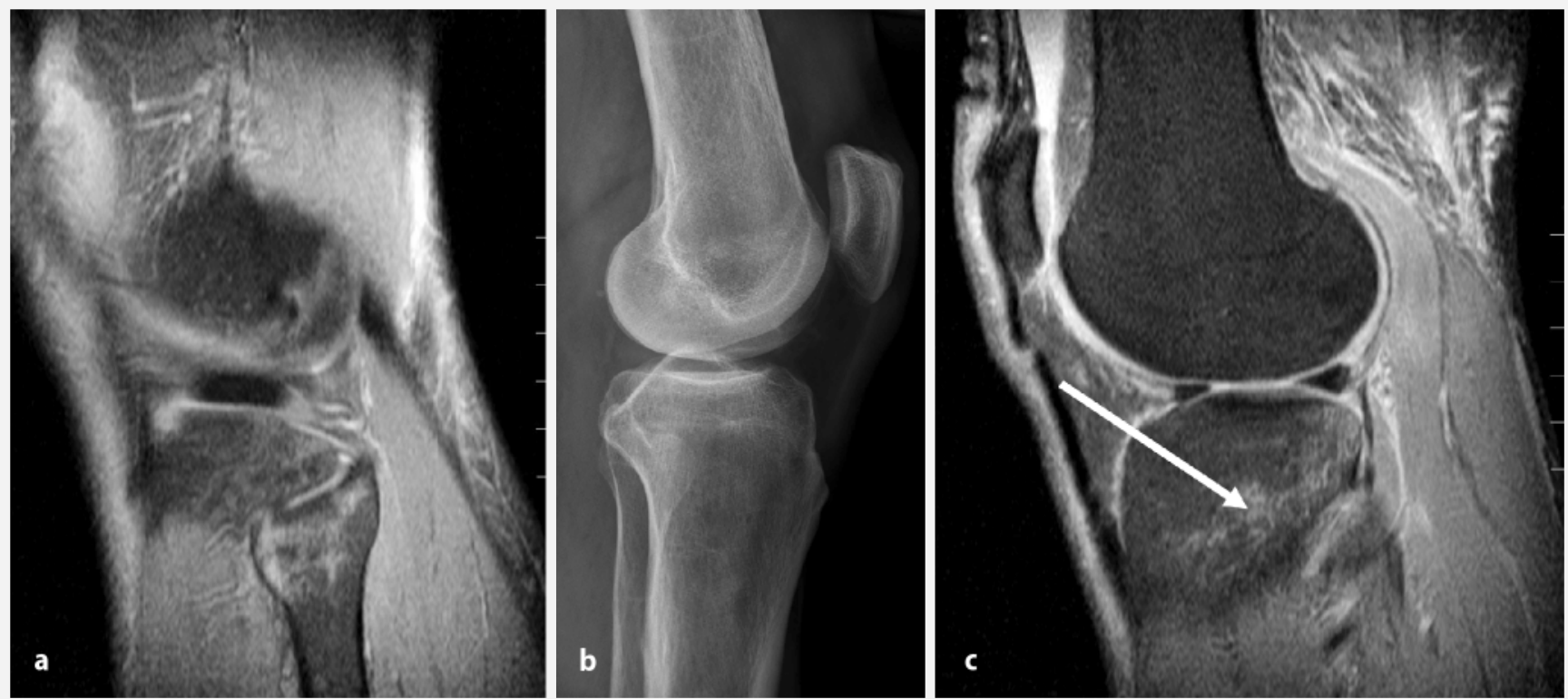

Abb. 5 \ Definition Bone bruise: seitliche Röntgenaufnahme des Kniegelenks (Projektionsradiographie, b) mit unauffälligem Befund, zusätzlich Darstellung protonengewichteter SPIR-Sequenzen (sagittal) der Fibula (a) und der dorsalen Tibia (c), Nachweis einer mäßigen Densitätserhöhung mit nicht geografischer Ausbreitung (Pfeil), Diagnose: Bone bruise (am ehesten Ödem)

Ihre klinische Wertigkeit erfährt die Bone bruise durch

a) weitere diagnostische Zeichen aus der Computertomo- und/oder Projektionsradiographie und

b) wesentlich durch ihre Einordnung in Anamnese und klinischem Verletzungsmuster („leeres Röntgenbild“ „auffällige Klinik").
Die Bone bruise ist keine Fraktur und muss von dieser abgegrenzt werden.

\section{Empfohlene MRT-Methode}

Nachfolgend sollen

- das MR-tomographische Erscheinungsbild,
- die Befundkonstellationen und -interpretationen sowie

- die klinische Einordnung

der Bone bruise erörtert werden.

\section{Periphere Gelenke/Knochen}

Klassischerweise werden 3 Sequenzen eingesetzt. Herauszuheben ist, dass sich eine 
Abb. 6 MRT-Befund, Erklärung für Unfallmechanismus: ausgeprägte ventralseitige Bone bruise des Femurs (MRT, b, Pfeil), Hinweis auf erhebliche Gewalteinwirkung, Befund zur klinisch und anamnestisch bekannten Patellaluxation korrelierend, röntgenologisch unauffälliger Befund bei erfolgter Reposition der Patella (a)

Abb. 7 Differenzialdiagnose und Kausa bei Mikrofraktur:T1-Wichtungen: Mikrofrakturen im Kalkaneus (Pfeil), zusätzlich Nachweis einer Dysplasie der Fettzapfen (a), vgl. regelrechten Be-

fund (b)

T1-gewichtete, eine $\mathrm{T}$ 2-gewichtete und eine protonengewichtete SPIR-Sequenz ergänzen sollten [3]. Die dabei gewählten Schichtorientierungen sollten in den 3 orthogonal zueinander stehenden Ebenen angefertigt werden. Auf diese Weise ist es möglich, einen Befund sowohl technologisch (T1-T2-STIR-Vergleich) als auch anatomisch in Beziehung zu setzen

(- Abb. 5, 6).

Grundsätzlich ist röntgenologisch und nachgeordnet magnetresonanztomographisch nach Verletzungsmustern im Gelenkbereich bzw. in den angrenzenden ossären Strukturen (z. B. Talus oder Kalkaneus oder Femurkondylus) zu suchen $[2,4]$. Eine Verwerfung von trabekulären Strukturen im Röntgenbild kann auf eine Mikrofraktur hinweisen (• Abb. 1, 7). Die Bone bruise ist hingegen definitionsgemäß im Röntgenbild nicht erkennbar. Wir
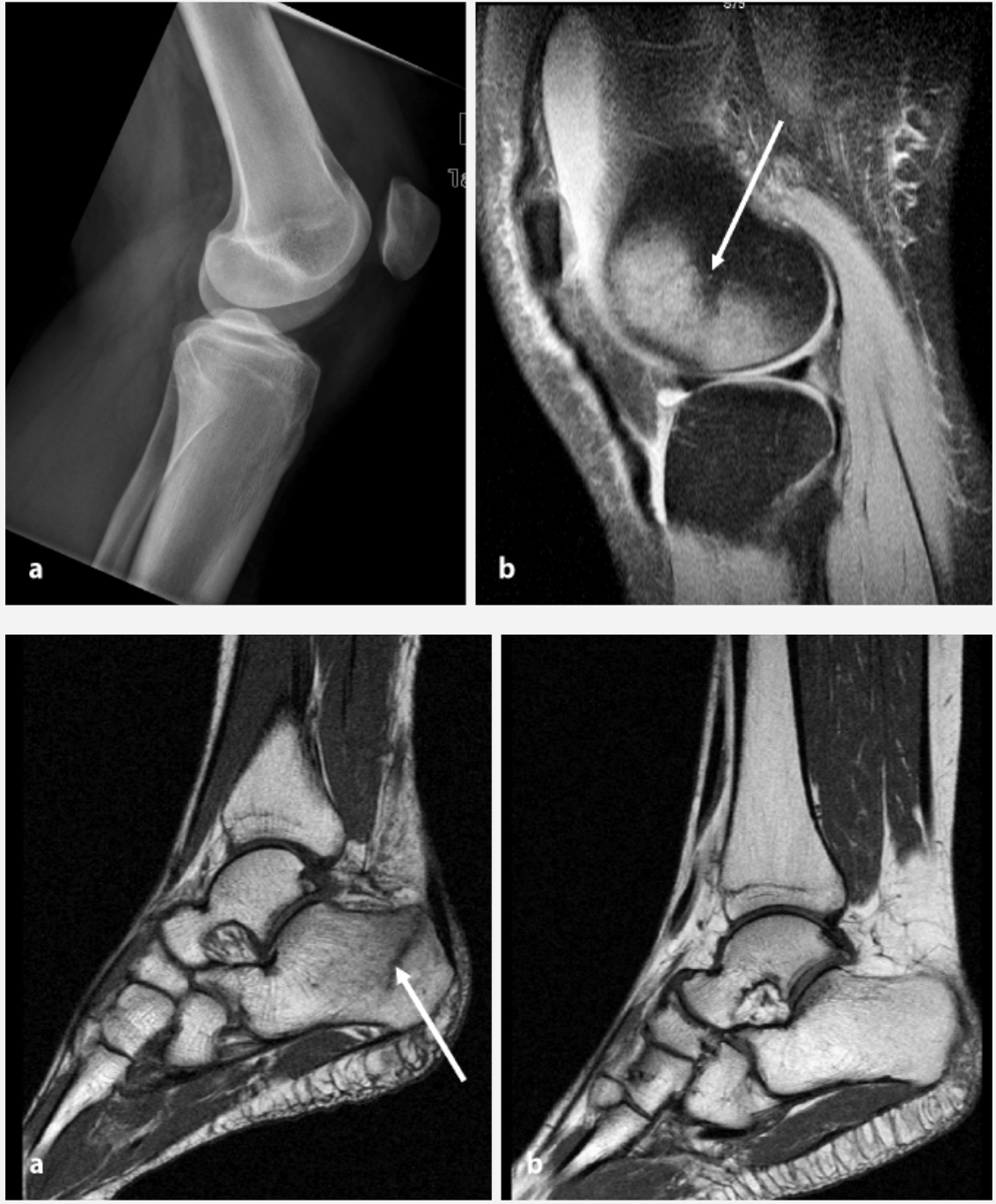

empfehlen dem strengen diagnostischen Prinzip - bei leerem Röntgenbild folgt Magnetresonanztomographie - zu folgen und in einer entsprechenden Befundkonstellation die MRT zu veranlassen.

Häufigste Manifestationsorte der Bone bruise sind

- im Handgelenkbereich die proximale Handwurzelreihe bzw. die Metaphysen von Radius und Ulna (• Abb. 8),

- im Bereich des Kniegelenks die dorsale Begrenzung der Femurkondylen bzw. vorderer oder hinterer Tibiarand (beachte v. a. das Sagittalbild) oder

- im Bereich des Talus die subchondralen kranialen Talusanteile bzw. der kaudale Anteil des Kalkaneus.

\section{Stammskelett}

Das Auftreten einer Bone bruise ist erwartungsgemäß im Stammskelett selten.
Infolge der geringeren Exposition ist es weniger prädestiniert, einem Mikrotrauma mit der Folge einer Bone bruise ausgesetzt zu sein. Verletzungen im Stammskelett sind in aller Regel Rasanzverletzungen, die in eindeutig projektionsradiographisch fassbaren Verletzungsmustern münden (Frakturen). Dennoch sind Bone-bruise-Befunde möglich.

Sinnvollerweise wird hier mit einer $\mathrm{T}_{1}$ , T2- und einer STIR-Sequenz in jeweils orthogonal aufeinander orientierten Ebenen untersucht. Insbesondere die $\mathrm{T}_{1}$ - und T2-gewichteten und hier vorrangig die fettgesättigten Sequenzen, erlauben folgende klinische Aussagen:

a) hoch sensitiver Hinweis auf Verletzungen - bis zu 96\% [1]

b) isolierte Nachweismuster (keine weiteren Verletzungsmanifestationen) erklären Schmerzkonstellation und be- 


\section{Bone bruise}
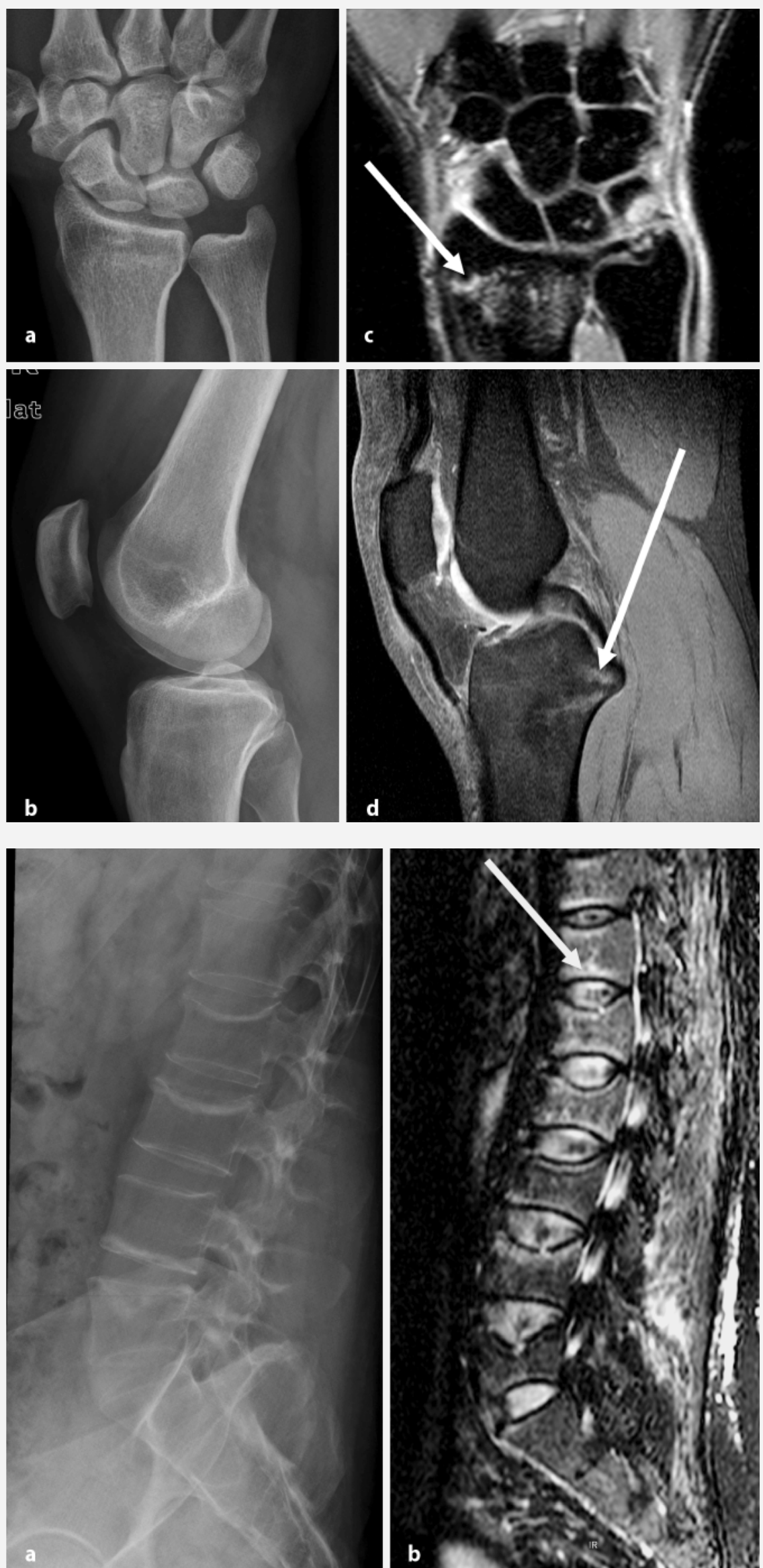

Abb. 84 MRT: hohe Sensitivität der SPIR-Sequenz: sowohl koronar (Hand) als auch sagittal (Knie) Nachweis nicht geografischer metaphysärer Veränderungen im distalen Radius bzw. in der Tibia (Pfeil, c, d), gegenüber projektionsradiographisch unauffälligen Befunden (a, b) Nachweis einer (morphologisch manifesten) Verletzung ohne Fraktur, cave: klinisches Beschwerdebild beachten - therapieführend

Abb. 94 Bone bruise an der Wirbelsäule: röntgenologisch Nachweis mäßiggradiger Sinterungen bei Osteopenie sowie subchondrale Sklerosierung (a), magnetresonanztomographisch in sagittaler Schichtorientierung hyperintense subchondrale Areale (Pfeil) (b), Befunde nicht den sklerosierten subchondralen Veränderungen der Degeneration entsprechend, eher Zeichen der noch persistierenden Sinterungsprozesse mit reaktivem Ödem 
gründen in aller Regel eine konservative Therapie

c) Sind keine Bone-bruise-Kriterien ableitbar, kann eine Verletzung ausge-

schlossen werden.

Grundsätzlich kann gesagt werden, dass mittels MRT frühzeitig ein Verletzungsmechanismus der Wirbelsäule ausgeschlossen werden kann. Dies hat insbesondere für spätere gutachterliche Beurteilungen bei Rentenbegehren oder der Bewertung von Folgebefunden wesentliche Bedeutung. Wir empfehlen daher dringend bei klinisch auffälliger Situation bzw. adäquatem Rasanztrauma, jedoch negativem Röntgenbild, die Anschlussdiagnostik mittels MRT (• Abb. 9).

\section{Auswirkungen der Diagnose Bone bruise}

Therapeutische Konsequenzen im Sinn eines chirurgischen Vorgehens werden aus der Diagnose Bone bruise in aller Regel nicht gefolgert. Hingegen ist ihr Nachweis für die Einordnung der Befunde aus chirurgischer Sicht ein wesentlicher Beitrag zur Klärung des Verletzungsmusters im Hinblick auf den Therapieansatz sowie die spätere Begutachtung [6].

\section{Fazit}

1. Ossäre und Weichteilverletzungen bedürfen einer subtilen Diagnostik mittels bildgebender Verfahren. Diese münden in differenzierte Therapieansätze.

2. Als Basis dient die Projektionsradiographie in 2 Ebenen. Ein im Hinblick auf Anamnese, Unfallhergang und klinischem Beschwerdebild adäquater Befundnachweis beendet in aller Regel die bildgebende Diagnostik.

3. Computertomographische Verfahren kommen als ergänzende Basisdiagnostik zur Beantwortung operationstaktischer Fragen zum Einsatz.

4. Bei diskrepanter Befundkonstellation zwischen auffälligem klinischem Befund, jedoch leerem Röntgenbild folgt unmittelbar die MRT zum Ausschluss von Mikrofrakturen bzw. Bone bruise.
5. Die Bone bruise ist eine an ein Trauma gebundene intraossäre Ödem- oder Hämatombildung. Sie kann hoch sensitiv mittel MRT erfasst werden. Sie ist gegenüber Mikro- oder okkulten Frakturen im Sinne der trabekularen Zerstörung abzugrenzen und mit diesen nicht gleichzusetzen.

6. Untersuchungstechnisch sind sowohl im Gelenk- als auch im Stammskelettbereich T1- und T2-gewichtete sowie STIR- und SPIR-orientierte Sequenzen einzusetzen. Es ist auf eine möglichst dreidimensionale orthogonale Darstellung zu achten.

7. Der Nachweis der Bone bruise indiziert in aller Regel kein aktives chirurgisches Vorgehen. Er dient jedoch der Einordnung des Verletzungsmechanismus, der Befundobjektivierung und ggf. der Auswahl des konservativen Therapieansatzes oder mittelfristigen Begutachtungen. Bei magnetresonanztomographisch fehlendem Nachweis einer Bone bruise ist eine Verletzung auszuschließen.

\section{Korrespondierender Autor}

\section{Dr. R. Braunschweig}

Klinik für bildgebende Diagnostik

und Interventionsradiologie,

BG-Kliniken Bergmannstrost, 06002 Halle, Saale

Rainer.Braunschweig@bergmannstrost.com

Interessenkonflikt. Keine Angaben

\section{Literatur}

1. Arndt WF, Truax AL, Barnett FM et al. (1996) MR-diagnosis of bone. AJR Am J Radiol 166: 119-124

2. Kaneko K, Demouy EH, Brunet ME (1993) Correlation between occult bone lesions and meniscoligamentous injuries in patients with traumatic knee joint disease. Clin Imaging 17: 253-257

3. Lal NR, Jamadar DA, Doi K et al. (2000) Evaluation of bone contusions with fat-saturated fast spinecho proton-density magnetic resonance imaging. Can Assoc Radiol J 51: 182-185

4. Mair SD, Schlegel TF, Gill TJ et al. (2004) Incidence and location of bone bruises after acute posterior cruciate ligament injury. Am J Sports Med 32: 1681-1687

5. Newberg AH, Wetzner SM (1994) Bone bruises: their patterns and significance. Semin Ultrasound CT MRI 15: 396-409

6. Stephenson T, Bialas Y (1996) Estimation of the age of bruising. Arch Dis Child 74: 53-55 